\title{
Simulation of Coupled Fire/Atmosphere Interaction with the MesoNH-ForeFire Models
}

\author{
Jean-Baptiste Filippi, ${ }^{1}$ Frédéric Bosseur, ${ }^{1}$ Xavier Pialat, ${ }^{1}$ \\ Paul-Antoine Santoni, ${ }^{1}$ Susanna Strada, ${ }^{2}$ and Céline Marii ${ }^{2}$ \\ ${ }^{1}$ SPE-CNRS UMR 6134, Campus Grossetti, BP 52, 20250 Corte, France \\ ${ }^{2}$ LA-CNRS UMR 5560, OMP, 14 Avenue Edouard Belin, 31400 Toulouse, France \\ Correspondence should be addressed to Jean-Baptiste Filippi, filippi@univ-corse.fr
}

Received 15 January 2011; Accepted 16 May 2011

Academic Editor: William E. Mell

Copyright ( $) 2011$ Jean-Baptiste Filippi et al. This is an open access article distributed under the Creative Commons Attribution License, which permits unrestricted use, distribution, and reproduction in any medium, provided the original work is properly cited.

\begin{abstract}
Simulating interaction between forest fire and atmospheric processes requires a highly detailed and computationally intensive model. Processing this type of simulations in wildland fires forbids combustion-based models due to the large amount of fuels to be simulated in terms of quantity and diversity. In this paper, we propose an approach that couples a fire area simulator to a mesoscale weather numerical model in order to simulate local fire/atmosphere interaction. Five idealized simulation cases are analysed showing strong interaction between topography and the fire front induced wind, interactions that could not be simulated in noncoupled simulations. The same approach applied to a real-case scenario also shows results that are qualitatively comparable to the observed case. All these results were obtained in less than a day of calculation on a dual processor computer, leaving room for improvement in grid resolution that is currently limited to fifty meter.
\end{abstract}

\section{Introduction}

Wildland fires are influenced by many physical processes, from which several of them directly stem from the atmosphere behaviour such as wind or humidity, showing a direct influence of the atmosphere on the fire. Feedback from the fire to the atmosphere has been studied and observed since the fifties [1], and several attempts to model and simulate fire-atmosphere interaction have been successful since then.

Among the most recent numerical studies of fire/atmosphere interaction, Mell et al. [2] have obtained with the wildland-urban interface fire dynamics simulator (WFDS) model a good correspondence between numerical results and real prescribed burning experiment of Australian grassland Cheney and Gould [3]. Similar numerical results were obtained by Linn et al. [4] using the HIGRAD/FIRETEC model performing several numerical investigations with different topography and wind conditions, but, unlike Mell et al. [2], no comparison to actual burns were made in these academic cases. These models focus on the processes of solid fuel pyrolysis, heat transfer, gas phase combustion, and local fire-atmosphere interaction that are essential to the physical mechanisms involves in fire spread. Nevertheless simulating these interactions at the scale of their appearance (i.e., the combustion scale) requires a highly detailed and computationally intensive model that is nowadays not reachable for actual wildland fires. Moreover, it is rarely possible to gather sufficient data to initiate a simulation at the level of detail required for such simulations.

On other hand, less physically detailed models based on the fire area simulator, such as FARSITE, are of a prime interest to the people who fight wildfires, and taking into account more of these coupled physical effects may permit to enhance the accuracy of such models.

The proposed approach has been developed to enable numerical fire/atmosphere coupling between available mesoscale atmospheric models (WRF, Meso-NH, etc.) with the family of fire area simulators. Numerical fire/atmosphere coupling has already undergone numerous studies, starting from the static fire simulations of [5] to more recent works where a simplified model of Rothermel type [6] fire spread is coupled with the so-called Clark-Hall atmospheric model [7] 
or the WRF mesoscale model [8]. While efforts at simulating coupled effects were fruitful even at the scale of large fires (several square kilometres), the use of Rothermel model may be subject to caution as effects of wind and slope on the rate of spread are expressed through coefficients that are experimentally fitted to wind values and usually uncorrelated. Moreover, the wind input into a fire area simulator, such as the Rothermel model, when used in a current operational setting, is almost always a near-surface single (temporal and spatial) mean wind provided by either a weather observing station (often hundreds of kms away from the fire) or predicted by a weather forecast model with resolution on the tens-of-kilometer scale at best. The operational forecast for surface fire propagation is, therefore, based on an input wind as if the fire was not here, that is, no local heterogeneous change in the wind field and fire behaviour due to the fire/atmosphere coupling can be taken into account.

In an effort to tackle these problems, a fire area simulator, named ForeFire, based on the propagation speed model of Balbi et al. [9] has been developed. In order to investigate fire/atmosphere coupling while aiming for operational ForeFire simulation code, it has been coupled with the Meso-NH model [10]. In an approach similar to Clark et al. [7], the mesoscale atmospheric model is coupled to a reduced front tracking wildfire model. This setup allows investigations on the differences induced by the atmospheric feedback in terms of propagation speed and behaviour. The main originalities of this combination resides in the fact that Meso-NH is run in a Large Eddy Simulation (LES) configuration and that the rate of spread model used in ForeFire provides a physical formulation to take into account effect of wind and slope.

\section{Numerical Models and Coupling Method}

In order to numerically couple the atmospheric and fire models, one has first to determine the physical phenomena responsible for the actual coupling.

Modelling the effects of the atmosphere on the fire (influence of the wind, humidity, etc.) represents a complex topic and has undergone a lot of studies. Modelling such a strong nonlinear dependence (moreover when slope is taken into account) has forced operational fire simulators such as Farsite to consider really simple models (usually, it is assumed that propagation velocity is linear with respect to the wind velocity normal to the front). This influence was also a challenge even for nonoperational research-oriented fire simulators fire simulators like FIRETEC or WFDS. In our case, these phenomena are embodied in the theoretical (and physicallybased) model of Balbi et al. [9] for the propagation speed that is presented hereafter.

Concerning the feedback from the fire on the atmosphere, one should take into account several phenomena such as heat transfer by means of convective heating and radiation and modification of the roughness of the canopy. In this first attempt to investigate the numerical coupling of both models solely energy fluxes from the fire front are taken into account as atmospheric model boundary conditions.
Still, the fire spatial scales are usually much lowers than the scale of resolution of the atmospheric (typically the order of hundreds-of-meter in our simulations). Thus, the front tracking method used to simulate the fire front needs a higher resolution than the atmospheric model.

2.1. Fire Propagation Model and Simulator. The rate of spread (ROS) model for the fire front (see, [9]) is based on the assumption that the flame is acting lake a tilted radiant panel heating the vegetation in front of it. It provides an analytical formulation of the propagation speed accounting for slope, wind speed, and fuel parameters effects. It belongs to the family of Rothermel-like models in the sense that the fire behaviour is only described by the mean of the propagation velocity of the fire front. Although more complete than the Rothermel formulation, several physical assumptions on the flow are made in order to derive the rate of spread $R$ in order to provide a computationally reachable for operational-use fire area simulator (unlike models solving the full NavierStokes equations like WFDS or FIRETEC).

Readers are referred to Balbi et al. [9] for full derivation of the model. For self-consistency, we will review here the major assumptions.

(i) Shape of the flame is assumed triangle with the base size on the ground given by the depth of the front in the normal direction.

(ii) Velocity in the flame is the geometric sum of the wind at the flame location and the buoyancy velocity.

(iii) Pre-heating is only induced by radiation (no heat convection).

(iv) Input air flow in the flame is supposed stoichiometric.

(v) Degradation kinetic is constant over time; that is, heat release from a flaming fuel is constant over the burning time $R_{T}$.

(vi) Propagation is normal to the existing front.

In the end, the model for the propagation speed of the front $R$ can be summarized in

$$
R=R_{0}+A \frac{R}{1+\left(R / r_{0}\right) \cos \gamma}(1+\sin \gamma-\cos \gamma),
$$

with $R_{0}$ the propagation speed in case of null wind and no slope (to be measured) and a the radiant coefficient.

The flame tilt angle relative to the ground normal $\gamma$ (which includes wind $U$, buoyancy effect $u_{0}$ and slope $\alpha$ ) is given by

$$
\tan \gamma=\tan \alpha+\frac{U}{u_{0}}
$$

Model parameters are either fitted, or can be deduced from fuel properties (see, Balbi et al. [9]). Given this velocity in supposedly each point of the fire front, a Lagrangian front tracking method is used for simulating the evolution of the fire front and, by the means of historical fronts, also the evolution of the burning area. 
ForeFire [11] simulation code uses the velocity model in order to integrate the front surface over time using a front tracking method. In this Lagrangian method, the fire front line is decomposed into a set of connected points or markers. According to the configuration of the two neighbouring markers, each marker is affected with a normal vector to the front pointing to the unburned material, as shown in Figure 1. As the front shape is represented as a polygon, the normal vector is approximated as the bisector angle. The front is propagating towards the outside of the polygon (in white in Figure 1), while markers are linked in the clockwise direction.

The velocity of each marker is given by the rate of spread model of Balbi et al. [9] and the direction that coincides with the normal to the fire front. This method has been selected due to its computational efficiency, and the ability to simulate the propagation of an interface at high resolution (less than one meter) needed to take into account different vegetations, roads, houses, and fire breaks over a large area typical of a wildfire accident (hundreds of square kilometres). Indeed, by advecting the markers by less than one meter at each step, the fuels seen by the markers can be spatially finegrained so as to have nonburnable areas such as roads or fire breaks. To estimate if a fire can or cannot pass a nonburnable area, fuel parameters are checked ahead of all fire marker along its normal at a distance equal to the front thickness.

The fire front thickness is constructed by looking into the history of the fronts. Each marker has a "parent" marker, and each parent keep in memory the time of ignition. With a simple tracing back of the parents till one is found to be completely burned (the current time is superior to the sum of the ignition time and the burning time $\tau$ ), one can find the rear of the fire area and thus the thickness of the fire front. It should be noted that this calculation of the front thickness is only an approximation of the theoretical front thickness in the direction of the normal needed in Balbi et al. [9], but allows for highly nonstationary effects when the fire front crosses discontinuities (in fuels, humidity, or topography, for example).

2.2. Meso-NH Atmospheric Model. Meso-NH is an anelastic nonhydrostatic mesoscale model [10] intended to be applicable to all scales ranging from large (synoptic) scales to small (large eddy) scales and can be coupled with an online atmospheric chemistry module. For the fire coupling application, Meso-NH is run in large eddy simulation configuration $(\Delta x \leq 50 \mathrm{~m})$ mode without chemistry. Turbulence parameterization is based on a 1.5-order closure [12], with a prognostic equation for turbulent kinetic energy in 3D. We selected open boundary condition for all tests. Momentum variables are advected with a centered 4th order scheme, while scalar and other meteorological variables are advected with a so-called monotonic piecewise parabolic method [13]. The externalised surface module SURFEX (aimed at providing physicallybased boundary conditions to Meso$\mathrm{NH}$ at ground level) is used for the fire feedback in the simulation.

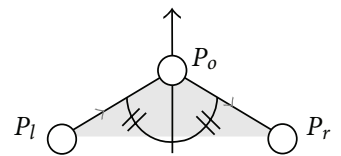

(a) (b)

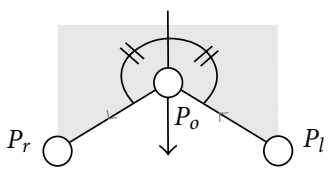

Figure 1: Front tracking and markers. Circles represent markers along the firefront line. Arrows show the propagation vector (bisector of the local angle at the marker $P_{0}$ between the point at left, $P_{l}$, and point at right, $P_{r}$ ). Grey area represents the burned fuel.

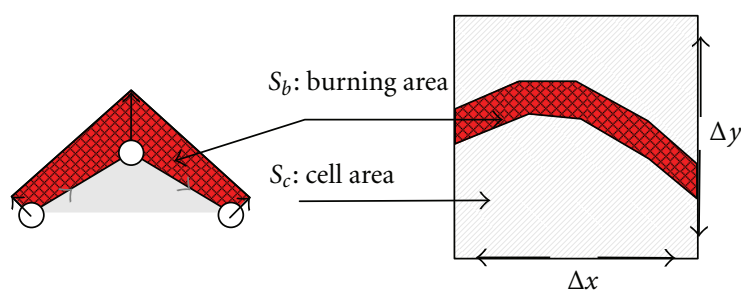

FIGURE 2: Integration of burning area. Red shape represents the fire front. Integration is performed on each atmospheric cell to compute the ratio of the burning area over the cell area.

2.3. Coupling Atmospheric and Wildfire Model. Finally, a specifically designed coupling component performs the simulation synchronisation, the data transformation, and interpolation.

The wildfire model acts in the atmospheric model as a new boundary condition, that is, injecting a heat flux $Q_{e}$ $\left(\mathrm{W} \cdot \mathrm{m}^{-2}\right)$, a water vapour flux $W v_{e}\left(\mathrm{~kg} \cdot \mathrm{m}^{-2}\right)$, and a radiant temperature $T_{e}(\mathrm{~K})$. Polygon clipping is used to derive the burning surface of an atmospheric cell (noted $S_{b}$ ) over the total cell area noted $S_{c}(\Delta x \Delta y)$ (Figure 2). The burning ratio for each atmospheric grid cell is noted $R b=S_{b} / S_{c}$.

As only a portion of the cell is burning, an equivalent radiant temperature for the whole cell is averaged from a nominal flame temperature $\left(T_{n}\right)$ and the soil temperature from the atmospheric model $\left(T_{s}\right) . T_{e}$ is given by

$$
T_{e}=\sqrt[4]{\left(1-R_{b}\right) T_{s}^{4}+R_{b} T_{n}^{4}}
$$

Equivalent heat fluxes corresponding to the energy of the hot gaseous column over an atmospheric cell is approximated from a nominal convective heat flux $\left(Q_{n}\right)$ with $Q_{e}=R_{b} Q_{n}$. Finally, equivalent water vapour fluxes, representing the amount of water vapour evaporated from the vegetation is interpolated over an atmospheric cell from nominal water vapour content $\left(W v_{n}\right)$ with $W v_{e}=R_{b} W v_{n}$.

$T_{n}$ is a fuel model parameter between 950 and $1100 \mathrm{~K}$ experimentally measured and different for oil/resin/lignin rich vegetation, and for all experiments, it has been set to $1000 \mathrm{~K} . T_{s}$ is the day temperature at the ground level. $W v_{n}$, is taken as the water content of the fuel per unit area.

The operation is performed for all atmospheric grid cells at ground level, that is, constructing three matrices that are passed to the atmospheric model as additional boundary conditions at the beginning of each time step of the atmospheric model. 
TABLE 1: Experimental parameters, with $A$ : Radiant factor, $R_{0}$ : rate of spread without wind and slope, $r_{0}$ flame thickness speed factor, $u_{0}$ : flame gas velocity, $R_{T}$ : fire residence time, $Q_{n}$ : nominal heat flux, $W v_{n}$ : nominal water vapor flux, and $T_{n}$ : nominal radiant temperature.

\begin{tabular}{cccccccc}
\hline$A$ & $R_{0}$ & $r_{0}$ & $u_{0}$ & $R_{T}$ & $Q_{n}$ & $W v_{n}$ & $T_{n}$ \\
\hline 1.5 & $0.1 \mathrm{~m} \cdot \mathrm{s}^{-1}$ & $0.01 \mathrm{~m} \cdot \mathrm{s}^{-1}$ & $5 \mathrm{~m} \cdot \mathrm{s}^{-1}$ & $30 \mathrm{~s}$ & $250 \mathrm{~kW} \cdot \mathrm{m}^{-2}$ & $0.1 \mathrm{~kg} \cdot \mathrm{m}^{-2} \cdot \mathrm{s}^{-1}$ & $1000 \mathrm{~K}$ \\
\hline
\end{tabular}

Concerning the effect of the atmosphere on the fire propagation, wind is interpolated in space using a bicubic method at the very location of the markers and in time by assuming the values of the wind, humidity, and all atmospheric variables to be constant throughout the atmospheric time step. All atmospheric model values are approximated from the first atmospheric level. Slope angle in the fire propagation direction is estimated from the elevation difference between the elevation at the fire marker and the elevation at the location projected after the estimated burning time $R_{T}$. Each elevation is also obtained a bicubic interpolation method.

\section{Idealised Experimental Setup}

In order to evaluate the ability of the proposed coupling approach and estimate the coupled influences of topography and wind on fire spread, five tests were run corresponding to a partial set of configurations proposed by Linn et al. [14] and solved by the same authors using fire spread model designed for smaller scales than the one presented here, thus making these simulations a reference for models designed for large wildfires. It should be pointed out that the configurations of Linn et al. [14] are idealized and were not compared to actual field observations. Given the paucity of observations in real-case scenario, the only available method of evaluation of models like $\mathrm{MNH} /$ ForeFire is the direct comparison with "reference" simulations such as the ones carried by Linn et al. [14].

The domain size is set to $640 * 320 * 500 \mathrm{~m}$ for all cases and discretized for the atmospheric model with a Cartesian grid whose parameters are a horizontal spacing of $16 \mathrm{~m}$ (in both direction of the ground) and an average vertical spacing of $20 \mathrm{~m}$. Boundary conditions were taken as open boundary conditions.

Base functions used to create the different topographies are taken from Linn et al. [14], which functions are used to create an idealized flat, canyon, hill ridge, and upcan terrains. In these simulations, the vegetation was modelled as a grass fuel bed with an inhomogeneous canopy with details as fine as discrete trees. As this level of refinement is not directly relevant to our propagation, model vegetation in our simulations is assumed homogeneous in the domain for all simulations. These values are based on mean values deduced from experimental studies [15] which exhibited rate of spread at flanks (relatively unaffected by wind or slope) close to the ones simulated by Linn et al. [14]. This resulted in an average dry fuel load of $7 \mathrm{~kg} \cdot \mathrm{m}^{-2}$ and parameters given in Table 1.

Atmospheric model background wind field is exactly the same for each case, with values of $6 \mathrm{~m} \cdot \mathrm{s}^{-1}$ constant in height. Ignition line in all cases is set to a 60 by 8 meters fire line located at the centre of the domain. A passive scalar tracer with a distribution set to the burning ratio of each grid point and for each atmospheric time step is used as a marker for smoke injection.

Figures 3, 4, 5, 6, and 7 present the simulation results for the flat, canyon, hill, ridge, and upcan cases $120 \mathrm{~s}$ after ignition. In all the figures, the red indicates the fire burning at the time of the snapshot, whereas the grey area represents the same front in an "uncoupled" simulation. The terms uncoupled stands for simulations without the twoway coupling of the fire and atmosphere considered here; that is, the atmospheric simulation is still performed, and winds at the Lagrangian marker location are still given by the interpolation of the atmospheric data. Slope is computed equally in each case, and thus, the only difference between the red and grey plots is taking into account the feedback of the fire on the atmosphere (influence of the atmosphere on the fire is modelled equally in both cases).

In the flat case (Figure 3(a)), the flow remains largely unaffected behind the fire. The simulation reveals an area of confluence ahead of the front with some recirculation that is located at the base of the fire plume (Figure $3(\mathrm{~b})$ ). The plume is relatively weak, affecting the flow to an altitude of $60 \mathrm{~m}$ over ground. Overall flow speed does not greatly differ from the original flow speed of $6 \mathrm{~m} \cdot \mathrm{s}^{-1}$. However, local enhancement of the surface velocity due to the coupling between the fire and the atmosphere leads to a greater ROS at the head of the fire compared to the noncoupling case. This effect can be attributed to the induced wind being taken into account in the coupled simulation.

The canyon case (Figure 4) clearly enlightens the strong influence of taking into account the coupling between fire and atmosphere in the simulation of the fire dynamics. In that case, the surface wind is strongly decreased in the canyon by topographic effects. These effects are not fully compensated by the increased slope, and we observe weaker ROS than in the flat case. In such scenario, the induced wind plays a major role in the dynamics of the fire spread, and the use of a coupled model results in increased ROS and better accounting of the physics.

With the same slope and same wind speed, the Hill case (Figure 5) presents a slightly different behaviour. The area of confluence is located here ahead of the fire front, so the maximum wind speed are just over the fire head. The resulting tilt angle results in a stronger ROS and a larger burning injection area. The effects of considering a coupling between the atmosphere and the fire are also of prime importance in the prediction of the rate of spread. In the hill case, the predictions issued by noncoupled simulations can be as low as half the rate of spread predicted during coupled simulations. 


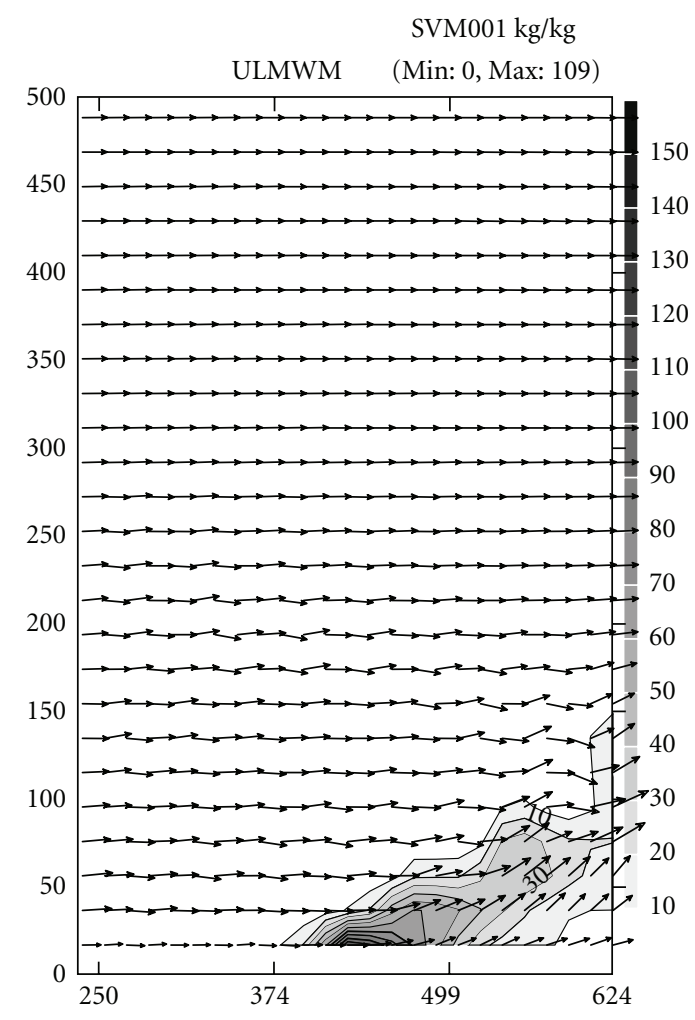

Time $=120$

$\rightarrow$ Minimum vector $4.3 \mathrm{~m} \cdot \mathrm{s}^{-1}$

$\longrightarrow$ Maximum vector $10 \mathrm{~m} \cdot \mathrm{s}^{-1}$

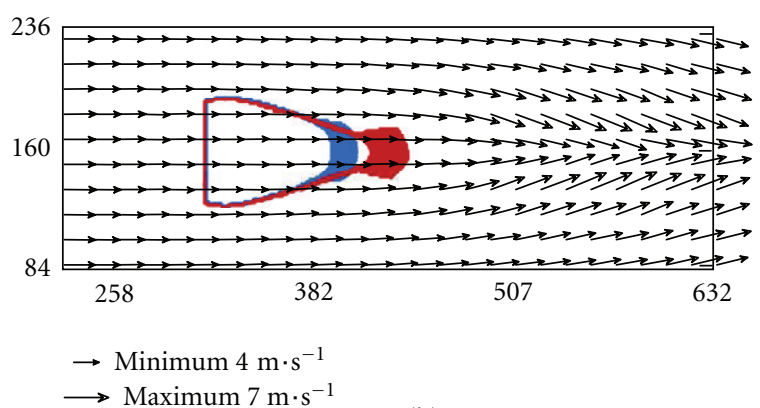

(b)

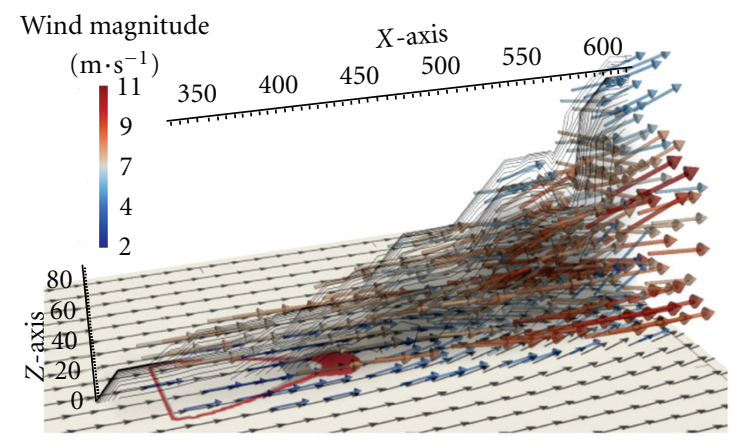

(c)

(a)

Figure 3: FLAT (a) Horizontal section $(x / y)$ at $Z=10 \mathrm{~m}$, fire lines after 120 seconds for the coupled (red) and noncoupled (grey) simulations. Arrows denote the wind vectors at ground level for the coupled case. (b) Cross-section $(x / z)$ of the coupled case at $Y=160 \mathrm{~m}$, shading represents concentration of the injected passive tracer. (c) $3 \mathrm{~d}$ wind field and passive tracer concentration isocontours.

Results for the ridge test case are presented in Figure 6. The topographic effects results in a widening of the burning area in the transverse direction of the wind due to slope gradient in that direction. In this case, the effect of taking into account the feedback from the fire on the atmosphere has less drastically changed the propagation speed of the front but still has a major influence on the depth of the fire front.

Results for the upcan test are shown in Figure 7. The narrowing of the fire head compared to the ridge case is of factor 3 in our case, whereas Linn et al. [14] results show a factor around 2. But in this case, simulating with a coupled approach provides much higher rates of spread than in the noncoupled simulation, accounting for the strong induced wind in the upcan.

Finally, Figure 8 presents for all cases the propagation distance of the fire front in the wind direction for three different types of simulations:

(i) results of Linn et al. [14] using FIRETEC, that is, where the fire propagation is resolved using a fullyresolving Navier-Stokes simulator, (ii) results of ForeFire for uncoupled simulations, that is, Rothermel-like propagation model with no feedback from the fire on the atmosphere is accounted for,

(iii) results of ForeFire for coupled simulations, that is, Rothermel-like propagation model with injection of heat, vapour, and passive scalar.

One can relate directly relate the rate of spread to the derivative of the propagation distance plotted in Figure 8, and thus make comparison on the behaviour of each model. As the FIRETEC simulations account for more physical phenomena than our simulation, it is assumed that the results of Linn et al. [14] represent the reference simulations.

Compared to FIRETEC results, uncoupled and coupled simulations both show an underestimation of the rate of spread in all cases. This is especially the case at the beginning of the simulations when the propagation model used in ForeFire is not able to capture the unsteady effects taking place in the transition regime. Though the underestimation can be partly imputed to the fuel properties which are different in our simulations and those of Linn et al. [14], 


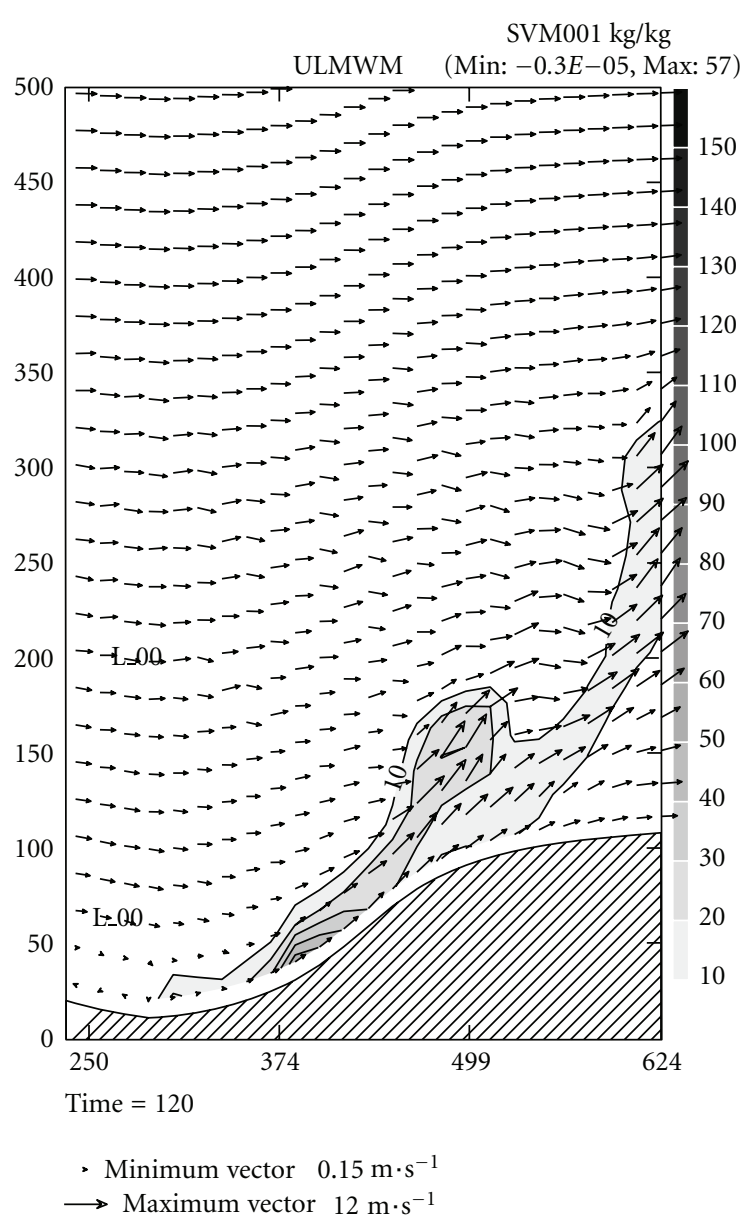

(a)

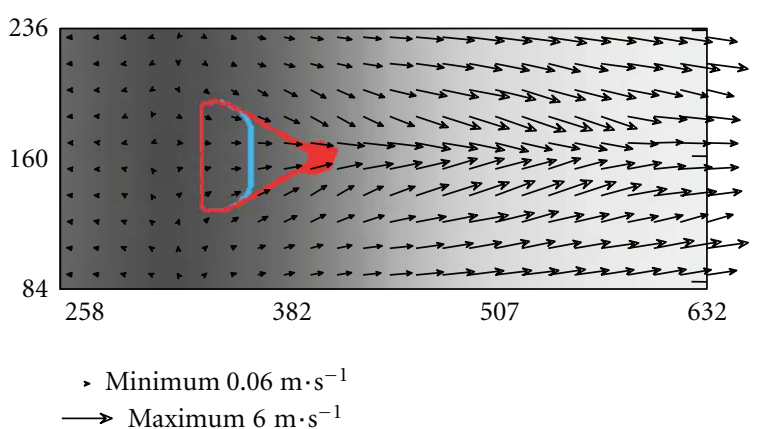

(b)

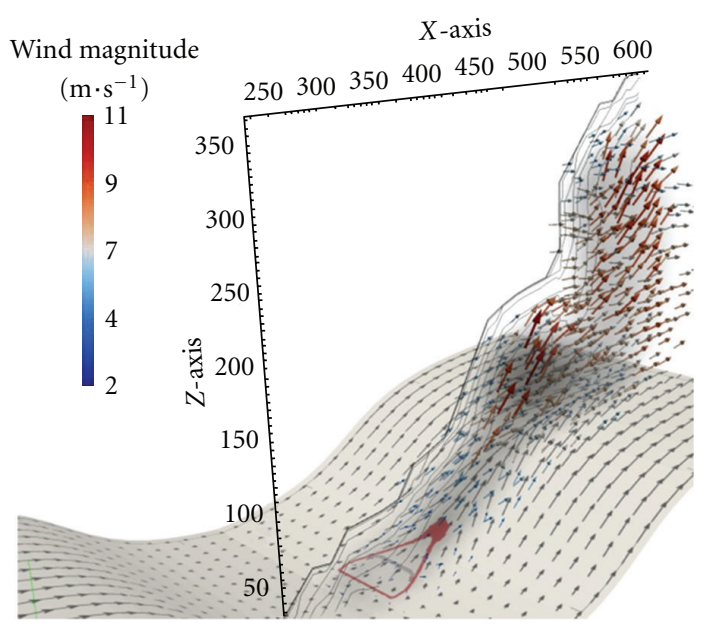

(c)

Figure 4: CANYON (a) Horizontal section $(x / y)$ at $Z=10 \mathrm{~m}$, fire lines after 120 seconds for the coupled (red) and noncoupled (grey) simulations. Arrows denote the wind vectors at ground level for the coupledcase. (b) Cross-section $(x / z)$ of the coupled case at $Y=160 \mathrm{~m}$, shading represents concentration of the injected passive tracer. (c) $3 \mathrm{~d}$ wind field and passive tracer concentration isocontours.

we decided not to change the parameters of the propagation model in order to assess only the effect of considering a full fire/atmosphere coupling.

Figure 8 shows that taking into account fire/atmosphere coupling always improve the results in terms of propagation distance. The term "improve" should be taken as "results are in better agreement with the reference simulation of FIRETEC". These improvements are of two types.

On one hand, the flat ridge and canyon cases exhibit only a quantitative improvement in the prediction of the propagation distance. Indeed, no change in the behaviour of the fire front is observed between uncoupled and coupled simulations. This is best seen in the canyon and flat cases where the propagation velocity tends to very low values whereas FIRETEC simulations do not show that kind of behaviour. These low values of rates of spread in our simulations are the consequences of a narrowing of the head front in our homogeneous ground-level vegetation, whereas in FIRETEC simulations, it is supposed that the fire propagates mainly in the crown.
On the other hand, in the hill and upcan cases, one definitely needs a coupled simulation in order to obtain subtle effects such as velocity enhancement by the fire and be able to predict plausible rate of spread. In these cases, simulations without fire/atmosphere coupling exhibit a different behaviour from the coupled ones as the rate of spread tends to very low values if uncoupled, whereas coupled simulations show rates of spread similar to the ones observed in Linn et al. [14].

While only comparing model to model, the level of accuracy (and computational cost) of both models are different and comparing results from a fire area simulator coupled to an atmospheric model such as ForeFire/MNH to a Navier-Stokes solver such as FIRETEC is once again a good way of assessing our model's results. As a consequence results show that taking into account fire/atmosphere coupling seems mandatory even in fire area simulator such as ForeFire. The authors believe this is a promising way of improvement of such simulators that should not be overlooked while fitting a Rothermel-like propagation model. 


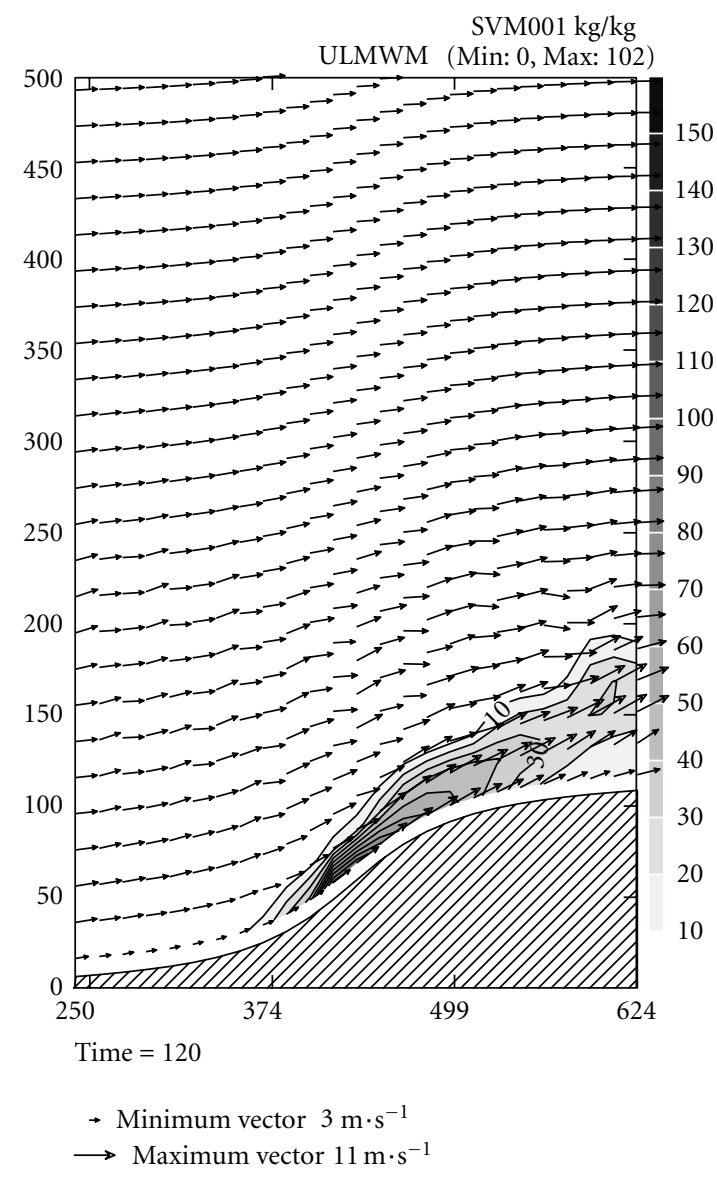

(a)

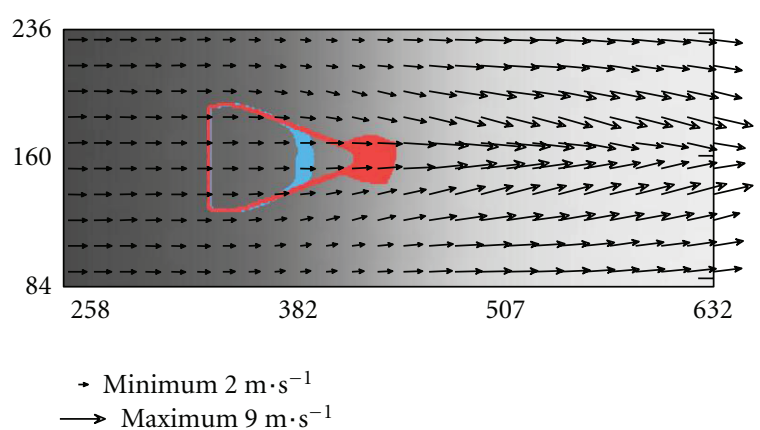

(b)

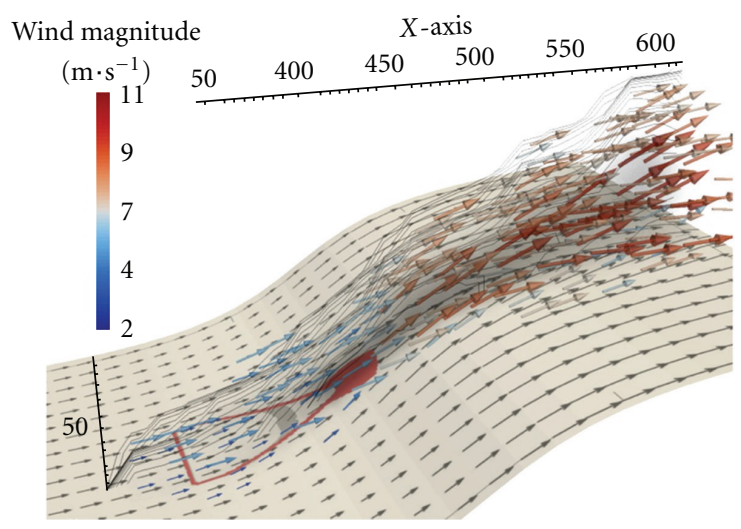

(c)

Figure 5: HILL (a) Horizontal section $(x / y)$ at $Z=10 \mathrm{~m}$, fire lines after 120 seconds for the coupled (red) and noncoupled (grey) simulations. Arrows denote the wind vectors at ground level for the coupledcase. (b) Cross-section $(x / z)$ of the coupled case at $Y=160 \mathrm{~m}$, shading represents concentration of the injected passive tracer. (c) $3 \mathrm{~d}$ wind field and passive tracer concentration isocontours.

\section{Real-Case Simulations}

The aim of coupling a fire area simulator (with underlying Rothermel-like model) to an atmospheric is to build a computationally affordable numerical tool for operational use while providing a frame for later improvements based upon physics. We then performed simulations of the coupled approach in two real-case scenarios (relatively welldocumented fires). These two fires occurred in the Corsican region, thus facilitating access to fuel data available.

4.1. Simulations Setup. The coupled simulations were run on a $2.5 \mathrm{~km} \times 2.5 \mathrm{~km} \times 1.5 \mathrm{~km}$ domain discretized on a $50 \times$ $50 \times 30$ mesh for the atmospheric model simulation $(\Delta x=$ $\Delta y=\Delta z=50 \mathrm{~m}$ ). Topography is given by the BDTOPO (IGN database) with a precision of $50 \mathrm{~m}$. Vegetation is extracted from the IFN database and classified between a homogeneous Mediterranean Maquis where fuel is present and nonburnable areas representing roads and buildings.

Both atmospheric conditions were initialized with radio soundings taken from the Ajaccio station at mid-day on the day of the fire (Figure 9).
Simulations were run on a Xeon $3.0 \mathrm{Ghz}$ processor (4 cores) for which it approximately takes 4 hours of simulation to obtain one hour of spreading in the real physical space. In those simulations, the fire propagation accounts for less than $5 \%$ of the total CPU time, and exact timing is difficult to point because the front tracking algorithm consumption depends of the number of markers in the simulation.

Vegetation in both simulations consisted of shrubs, similar to the fuel model described in [15]. The only differences with model parameters of Table 1 is the vegetation water content, that is reflected by a larger $R_{0}$, water vapor emission, and a lower $u_{0}$. For both cases, vegetation water content was assumed to be similar, as it corresponds to three consecutive days without rain, reaching ambient humidity $(60 \%$ relative air humidity in both cases) (Table 2).

Case 1 (Vazzio). The Vazzio fire occurred on the 16th of October 2007 near. The fire ignited around 14:30 on a day with stable and dry meteorological conditions, with a ground temperature of 20 degrees. The radiosounding made at the airport about three kilometers away at 12:00 gives a sustained westerly wind of about 4 to $5 \mathrm{~m} \cdot \mathrm{s}^{-1}$ with gusts of about the 


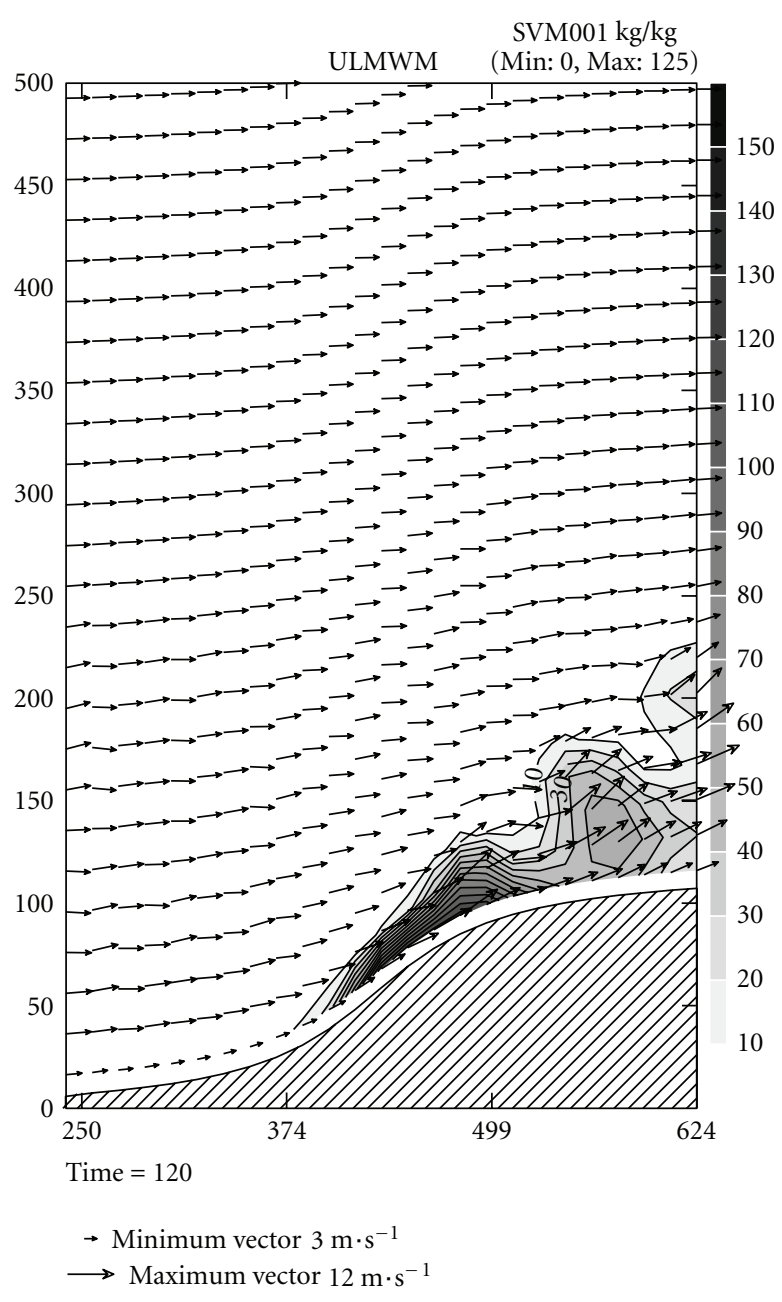

(a)

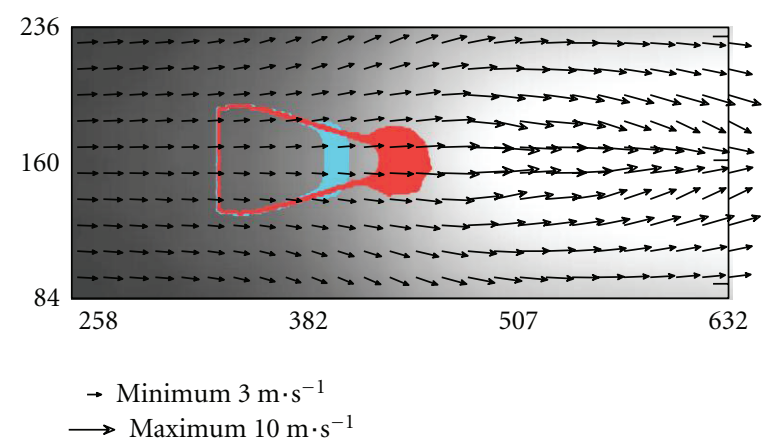

(b)

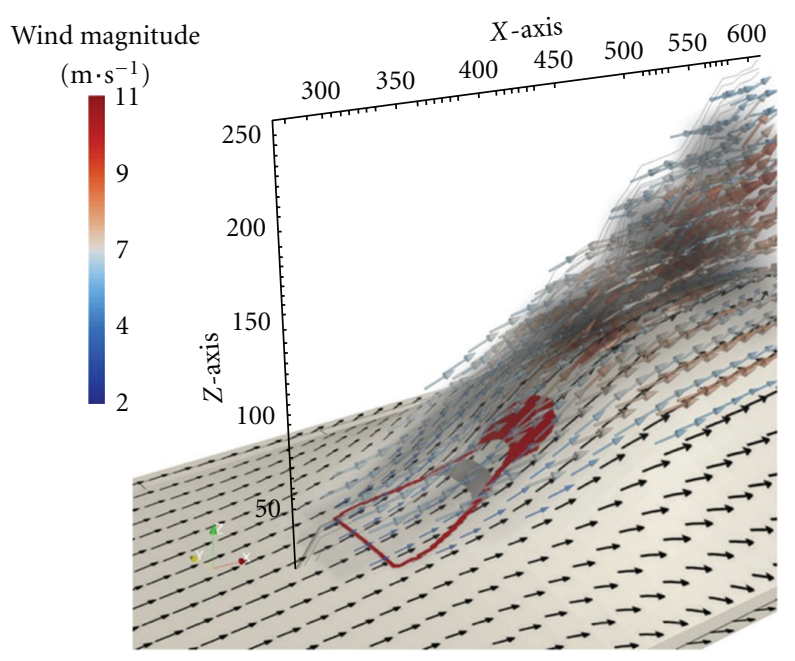

(c)

Figure 6: RIDGE (a) Horizontal section $(x / y)$ at $Z=10 \mathrm{~m}$, fire lines after 120 seconds for the coupled (red) and noncoupled (grey) simulations. Arrows denote the wind vectors at ground level for the coupledcase. (b) Cross-section $(x / z)$ of the coupled case at $Y=160 \mathrm{~m}$, shading represents concentration of the injected passive tracer. (c) $3 \mathrm{~d}$ wind field and passive tracer concentration isocontours.

TABle 2: Experimental parameters, with $A$ : Radiant factor, $R_{0}$ : rate of spread without wind and slope, $r_{0}$ flame thickness speed factor, $u_{0}$ : flame gas velocity, $R_{T}$ : fire residence time, $Q_{n}$ : nominal heat flux, $W v_{n}$ : nominal water vapour, flux and $T_{n}$ : nominal radiant temperature.

\begin{tabular}{cccccccc}
\hline$A$ & $R_{0}$ & $r_{0}$ & $u_{0}$ & $R_{T}$ & $Q_{n}$ & $W v_{n}$ & $T_{n}$ \\
\hline 1.5 & $0.12 \mathrm{~m} \cdot \mathrm{s}^{-1}$ & $0.01 \mathrm{~m} \cdot \mathrm{s}^{-1}$ & $4 \mathrm{~m} \cdot \mathrm{s}^{-1}$ & $30 \mathrm{~s}$ & $250 \mathrm{~kW} \cdot \mathrm{m}^{-2}$ & $0.03 \mathrm{~kg} \cdot \mathrm{m}^{-2} \cdot \mathrm{s}^{-1}$ & $1000 \mathrm{~K}$ \\
\hline
\end{tabular}

same magnitude. The wind changed direction during the event, but as no radiosounding are available to account for wind changes, the simulation was run with only the westerly forcing wind. The fire experienced almost free propagation till 15:40 and was finally stopped around 18:30 and was fought mainly over the head of the front by air attack. It burned up to $0.60 \mathrm{~km}^{2}$ of land with the burned area contour reported in Figure 10.

Case 2 (Favone). The Favone fire occurred on the 8 th of July 2009 near the village of Favone (south east Cosica). The fire was detected at 15:00 and experienced almost free propagation till 16:30 under a sustained and whirling wind of about 4 to $5 \mathrm{~m} \cdot \mathrm{s}^{-1}$. The fire was fought for protection along its flanks, passed the road at 16:00 and arrived to the sea at 16:15. Total extinction of the fire was declared at 19:00, with a total burning area of 25 ha. As in the Vazzio case, direct comparison between simulations and observations are to be handled with care as no fire fighting is taken into account in simulations (taking the fire fighting into account is possible, but information about fire fighting is scarce).

As for the first case, atmospheric conditions were stable and dry with a ground temperature of about 27 degrees and a west westerly wind of about $5 \mathrm{~m} \cdot \mathrm{s}^{-1}$.

4.2. Results and Discussion. For the selected fires, it was not possible to gather specific quantitative measures over the fire plume (such as plume height or smoke concentration at 


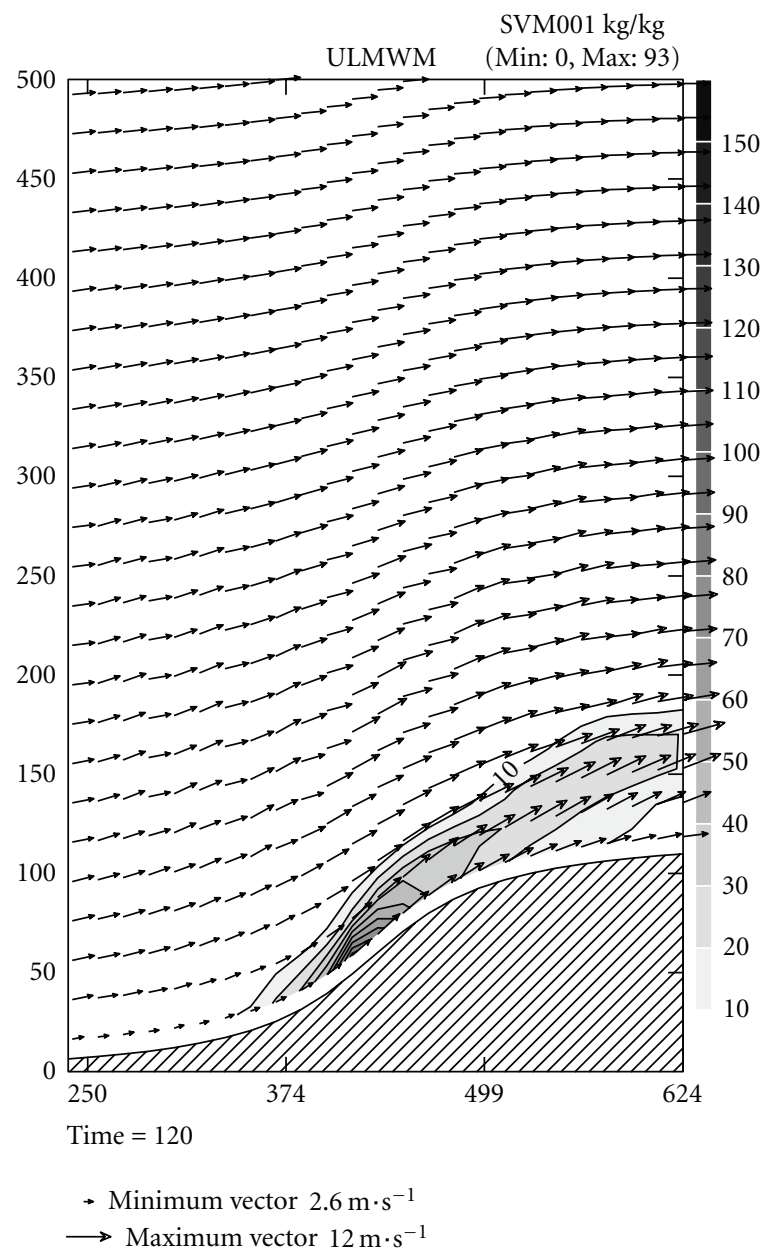

(a)

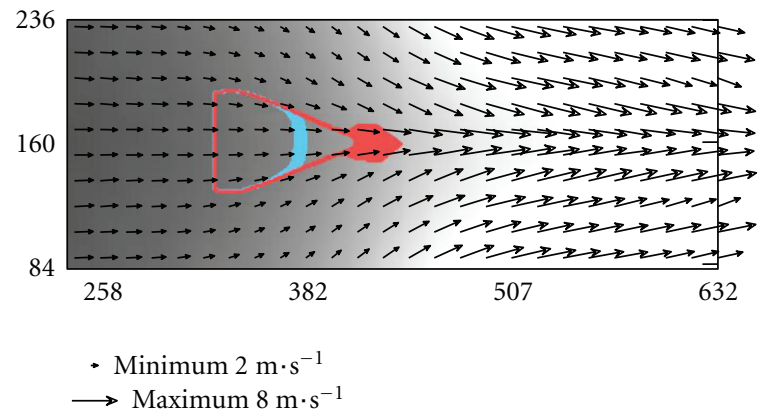

(b)

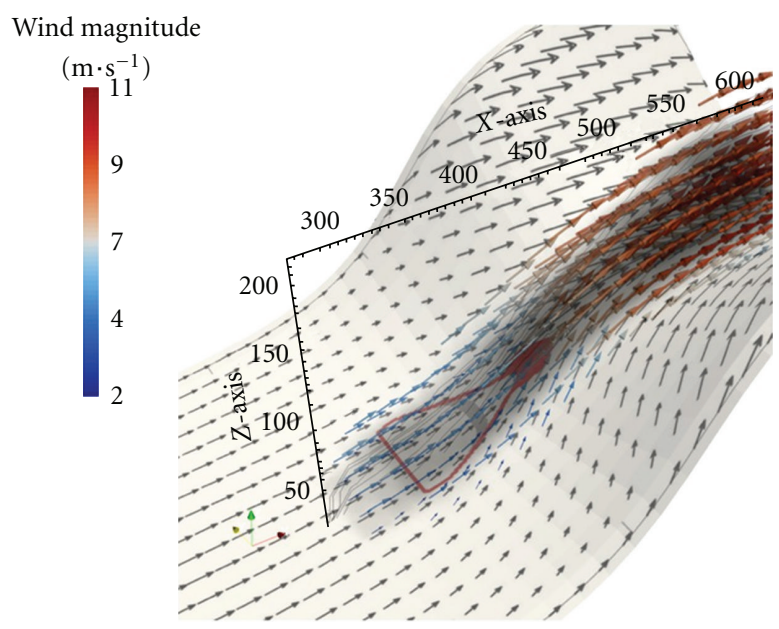

(c)

Figure 7: UPCAN (a) Horizontal section $(x / y)$ at $Z=10 \mathrm{~m}$, fire lines after 120 seconds for the coupled (red) and noncoupled (grey) simulations. Arrows denote the wind vectors at ground level for the coupledcase. (b) Cross-section $(x / z)$ of the coupled case at $Y=160 \mathrm{~m}$, shading represents concentration of the injected passive tracer.

specific points); nevertheless, a qualitative analysis is possible since some pictures were taken during those fires, thus enabling some qualitative verification. As the goal of the simulation is to be used as a decision support system, a satisfying verification would be to compare the general aspect of the plume as well as the modification of the wind field that affects the front. For all simulations, smoke concentration is given in unit $\cdot \mathrm{m}^{-3}$ and corresponds to the passive scalar tracer, with one unit being injected every second per mater square. Wind is provided in $\mathrm{m} \cdot \mathrm{s}^{-1}$.

A general behavior observed in both cases is the separation of the plume in two distinct area, the first one (from the front to about 500 meters) is the strong convective column and a second one, more diffuse, where the atmospheric flow is transporting the smoke into the atmosphere.

Results are in qualitative agreement with this behavior in both cases (Figures 10 and 11). In Case 1, the first part of the fire plume appears as a concentrated, opaque, and thick area of smoke that becomes suddenly diffuse. In Case 2 (Figure 11), both parts are separated, with the upper part changing direction while catching higher atmospheric winds. Both changes in direction and shape are in accordance with the tephigrams (Figure 9), where a ground atmospheric layer of about 100 meters high can be observed as a faster drop in temperature.

Another observation that can be made on Figure 11 (Case 2) is the initial separation of the plume into two parts, one on each flank of the fire. From the picture, we can note that the two flanks are generating two plumes that are later rejoined just over the most active front of the fire. Simulation well reproduced this behavior with the same initial separation of the plume that are merging while on top of the most active part of the front. While these two convective columns appears to be clearly separated, it is difficult to analyze and draw a picture of the flow along the columns and distinguish clear contrarotative behavior as simulation outputs at discrete time steps mainly represent eddies moving along these columns.

Figure 12 shows a thick plume that is transported over the sea with very little smoke reaching the shore. Similar 


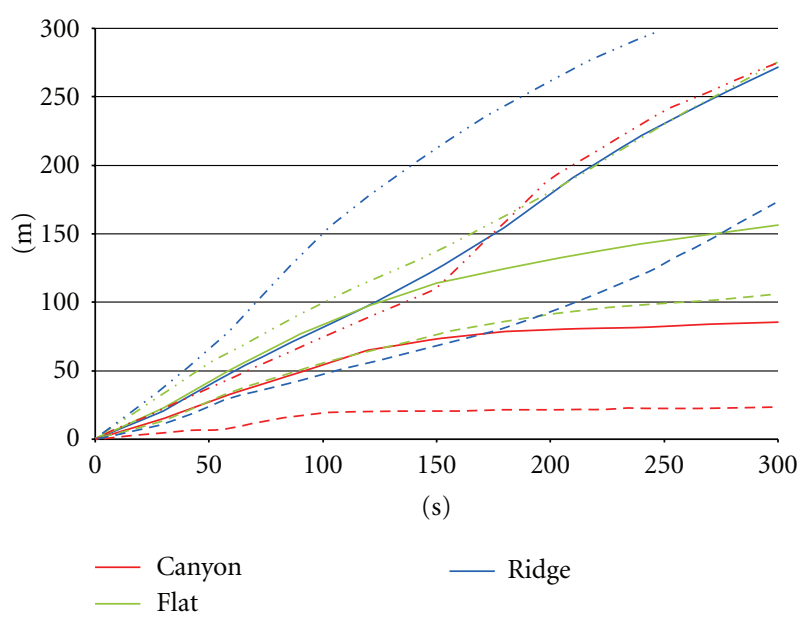

(a)

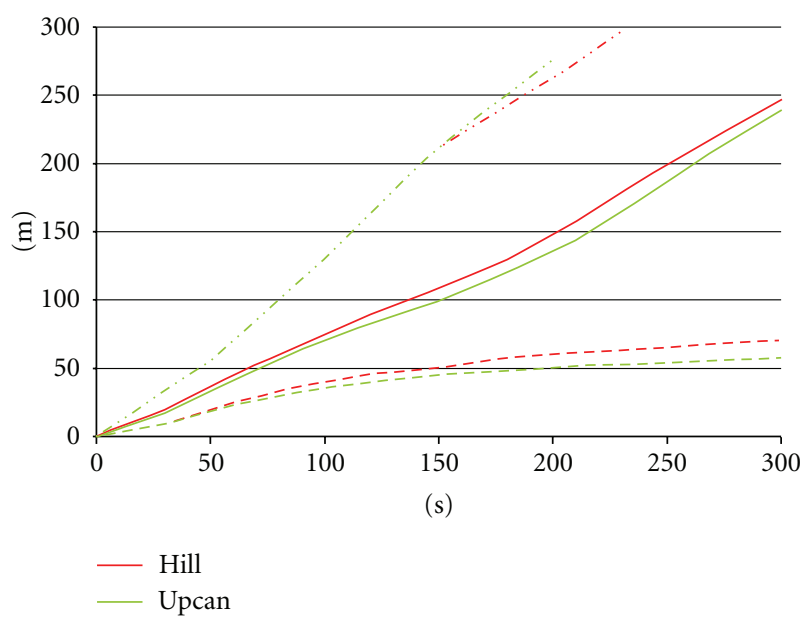

(b)

FIgURE 8: Propagation distance of the fire front function of time for the Canyon, Flat, Ridge cases (a) and Upcan, Hill cases (b). Firetec results are represented in dashed-dotted lines, while uncoupled results are shown by dashed lines and coupled results in plain lines.

behavior is observed in the simulation, with the front arriving approximately at the same time over the road as a thick, well-formed plume. Nevertheless, it appears that the angle between the plume plane and the sea plane is not well represented, but without knowing the exact time of the picture or the time of ignition, it is not possible yet to use this observation as a qualitative measure for the validation.

For all simulations, the structures of the simulated plume are not as refined as in the real one, but this is mainly due to the relatively low refinement of the grid for the atmospheric simulation $(50 \mathrm{~m})$. Simulated direction and height of the plume are similar to the observed ones. Nevertheless, dispersion seems to be underestimated in our simulation as the plume expansion is slightly lower in the simulation. This drawback supposedly mainly stems from the coupling fluxes injected by the fire simulation. As explained earlier in this paper the forcing fluxes from the fire are the heat flux, the flux of water vapor and the radiant temperature. Thus, no turbulent kinetic energy is directly injected in the atmospheric simulation, and thus, the fine structures of characteristic length less than $50 \mathrm{~m}$ (observed in the fire and assumed to contribute to the agitation of the atmosphere) are not taken into account in the present coupling.

Figures 13 and 14 present the intermediate and final front shape for Cases 1 and 2. One major feature of the proposed model is the ability to simulate topographic effects such as fire confinement by crests. In Figure 12 (Case 1), we can observe that the simulated contours reported in Figure 12 are in better agreement with the observations concerning the north side of the fire front, where changing slope effects have maintained the fire on one side of the hill. With a constant, noncoupled wind field, the simulated front is passing over the hill, which is less in accordance with the observed fire.

A major effect of the coupled wind field for Case 2 (Figure 14) is the acceleration near the ignition point. A direct consequence of this wind acceleration is that the backfire is propagating much slower, with a better accordance with observation.

Nevertheless, a side effect of the wind acceleration near the front in coupled simulation is to constrain the front on the flanks. For both cases, it appears that coupled simulation does underestimate the side propagation of the fire; it is particularly true in the Favona fire (Case 2), as this fire was fought on its flanks and is still underestimated by the simulation.

Plotting the general surface wind field for both simulations is not possible due to the fact that fields from the coupled simulation are dynamic and constantly changing during the simulation.

\section{Conclusions}

In order to be able to simulate subtle but nonetheless important physical phenomena such as induced wind or smoke dispersion, a coupled model has been developed synchronising the MesoNH atmospheric model with the physically based Lagrangian front tracking ForeFire wildfire simulator. With a straightforward coupling method, the atmospheric model is able to simulate the atmosphere dynamic induced by the fire and the subsequent effects on the RoS with meaningful results.

The five idealized scenarios allowed simulating induced flow patterns similar to those observed from simulations done by Linn et al. [14] with HIGRAD/FIRETEC. Transverse topological effects seem to be of more importance in our model as the widening/narrowing of the head fire is significantly greater in our simulations. The main feature of these simulations still remains that the fire head spread rate in the wind direction exhibits similar behaviours to those found by Linn et al. [14] in coupled simulations. The use of such fire/atmosphere coupling is mandatory in two of the five cases to retrieve behaviours similar to those simulated with FIRETEC. Results show large improvements in the prediction of the propagation distance along the wind direction for all cases when using coupled models. Rates of spread are still underestimated but show a much stronger qualitative 


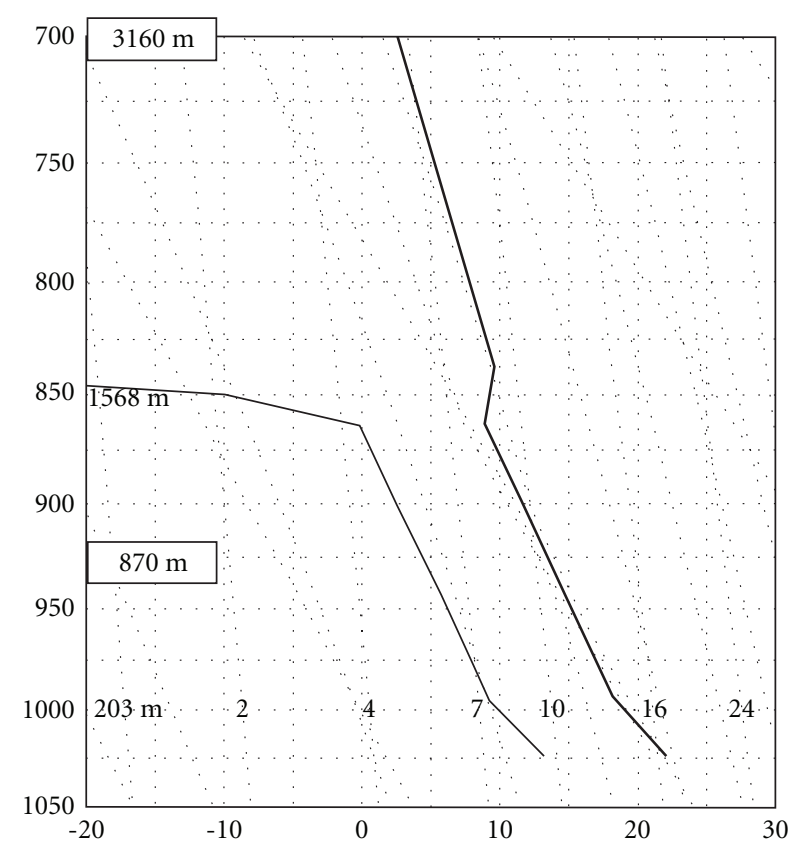

(a)

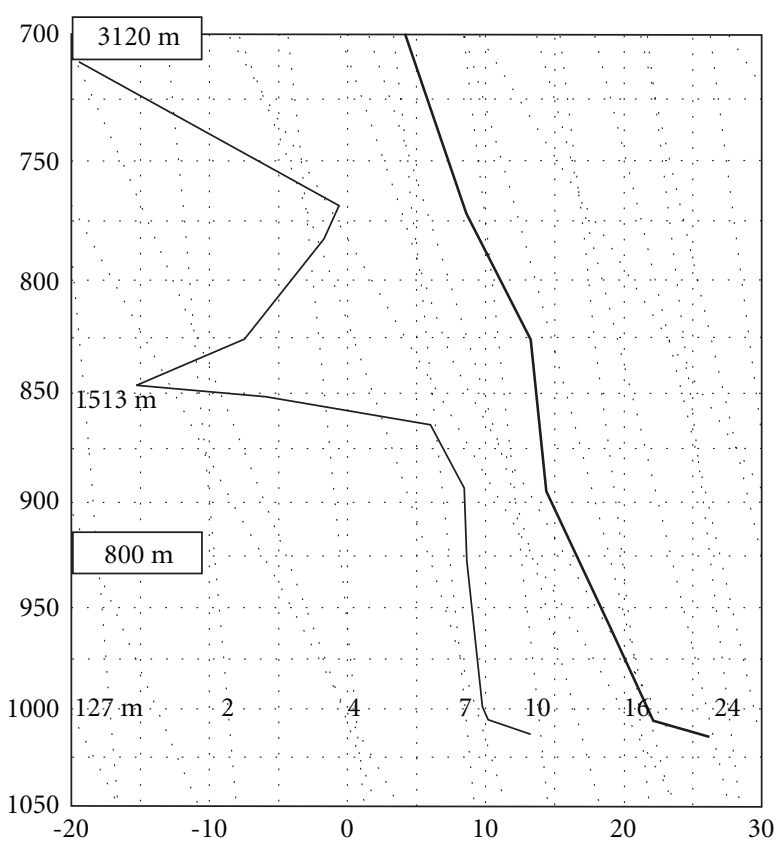

(b)

Figure 9: Tephigrams to $700 \mathrm{mb}$ for Case 1 (a) and 2 (b). Thicker line represent Temperature and thinner line the dew-point temperature. Wind profile for each case is represented by wind arrows on the right side of each graph.
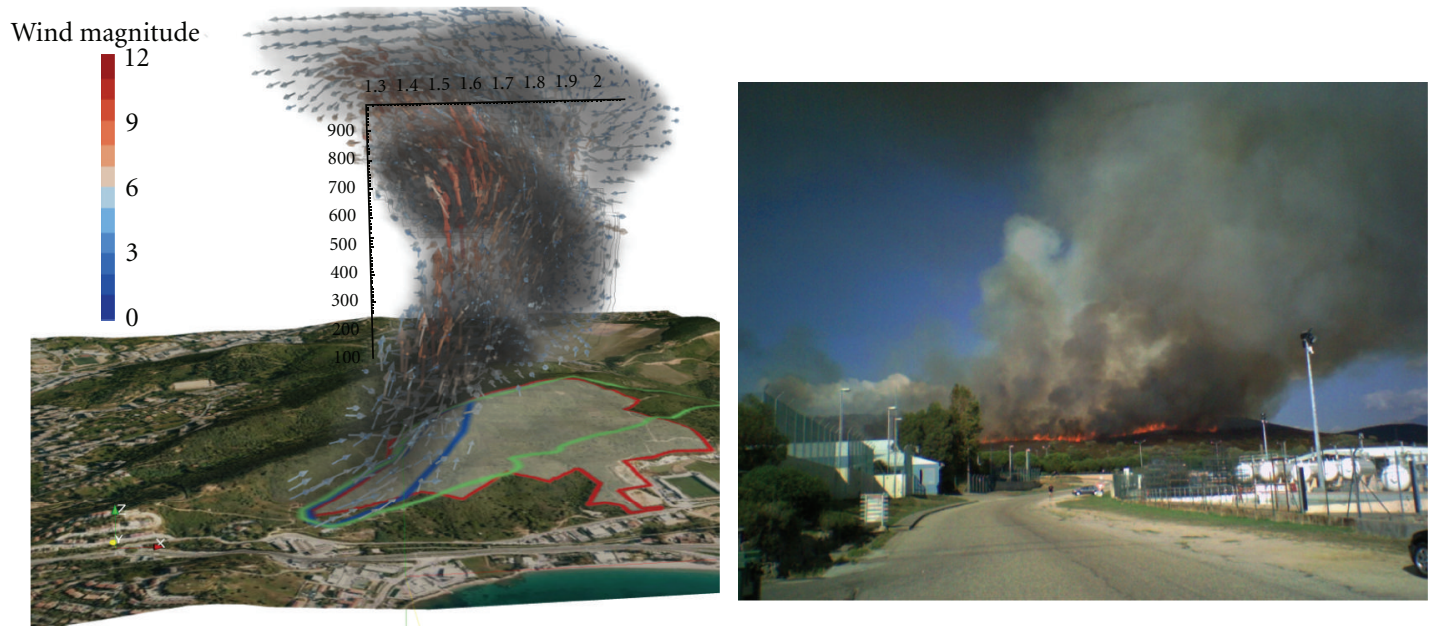

FIGURE 10: Case 1: Simulated and observed plume. Simulated plume is given $1 \mathrm{~h}$ after the fire ignition (the blue contour represents the fire front at that time) as the observation was taken approximately at the same time.

agreement with the reference simulations. This behaviour is of particular interest, as performing HIGRAD/FIRETEC simulations of the flow and fire patterns over a complex vegetation distribution with high resolution is nowadays computationally unreachable for large-scale wildland fires.

The proposed coupled model was then applied to two real-case scenarios and compared with observations. Model's behaviour is qualitatively similar to the real fire in simulating the fire propagation in terms of plume behaviour, with apparent plume similarities based on pictures taken the day of the actual fire. Nevertheless, while the front velocity formulation used in this study was not built to use input wind "as the fire was not there", it is still remains a rather parametric model that must be enhanced.

The objective in this paper was to move from fire area model with forced wind fields to coupled wind field that could represent the local perturbations affecting fire behaviour. As such, and considering the relatively small computational time (few hours for a medium size fire on a small cluster), these simulations seem to provide yet a good insight in terms of plume behaviour and fire wind effect. 

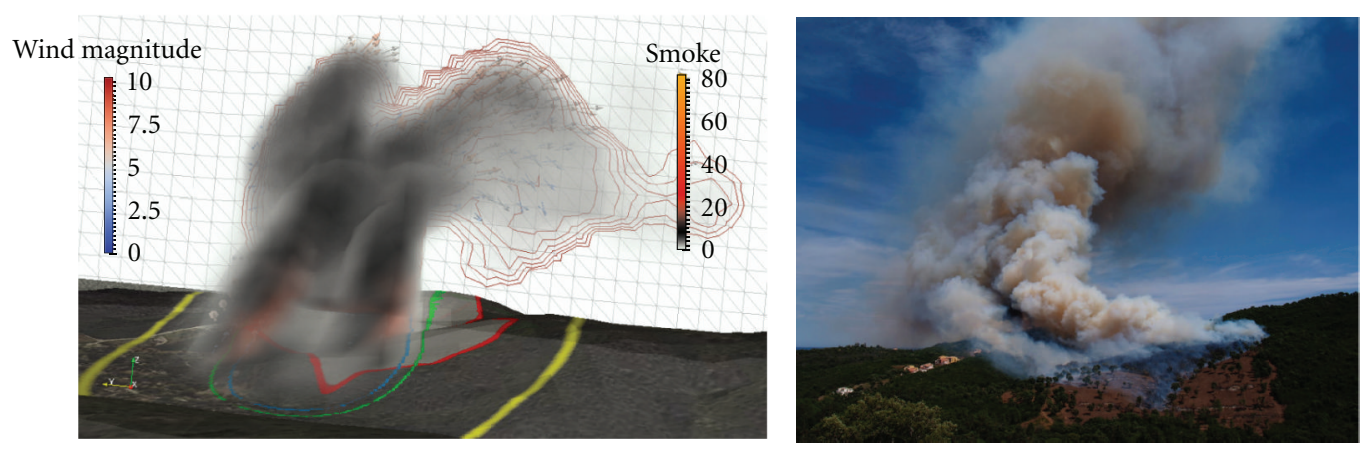

FIGURE 11: Case 2: Simulated and observed plume. Simulated plume is given 50 minutes after ignition (the blue contour represents the fire front at that time) with observation taken approximately at the same time.
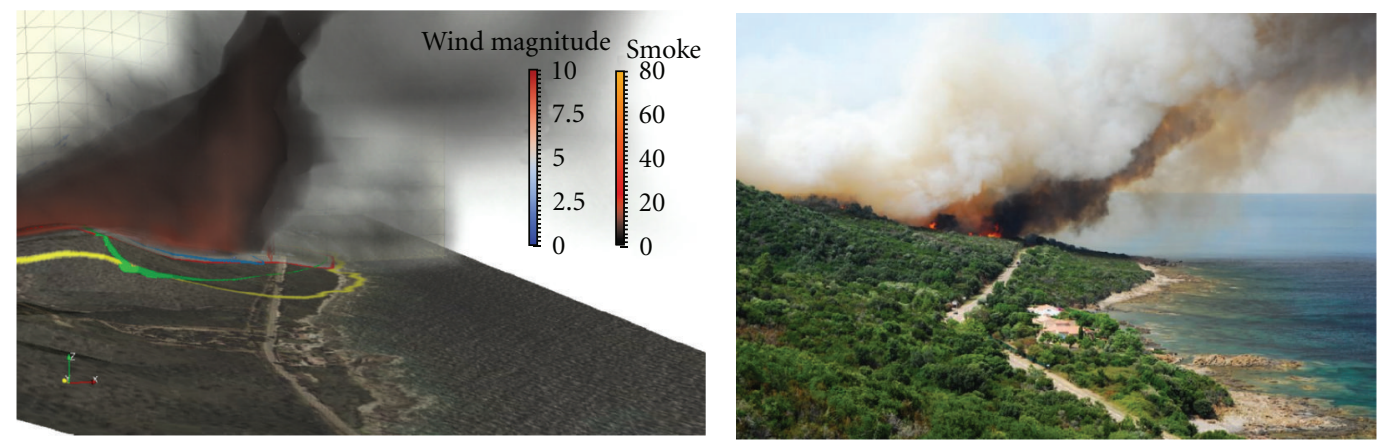

FIGURE 12: Case 2: Simulated and observed plume. Simulated plume is given 50 minutes after ignition (the blue contour represents the fire front at that time) with observation taken approximately at the same time.

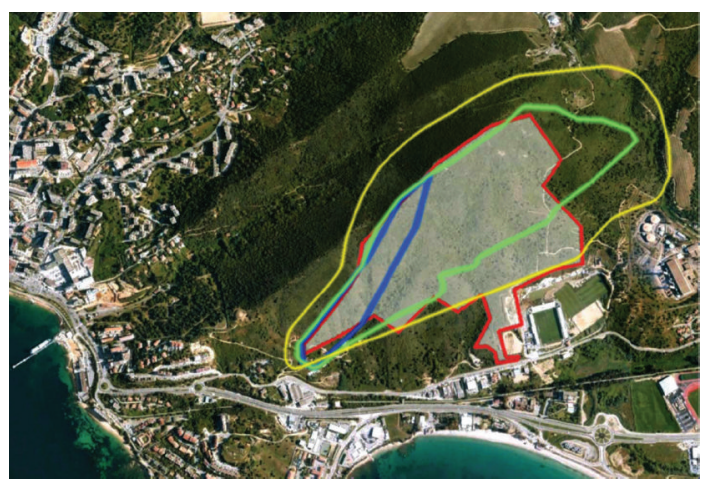

Figure 13: Case 1: Simulations results and observations for the Vazzio fire. Blue: simulated fire contour at 15:30 (after 1 h), Green: simulated contour at 18:30, Yellow: Simulated contour at 18:30 (non coupled); Red: final observed contour of the fire.

As a decision support tool, coupled simulation may help to forecast plume size, transport dispersion and smoke concentration at the ground, information of prime importance to protect the population, and anticipate the visibility loss for the fire fighters and civil transport in general.

More work is now carried out on the forest fire propagation code in order to use a better, nonparametric, description of fire fuels. Further enhancements are also planned to perform simulation of large past fire and simulation with

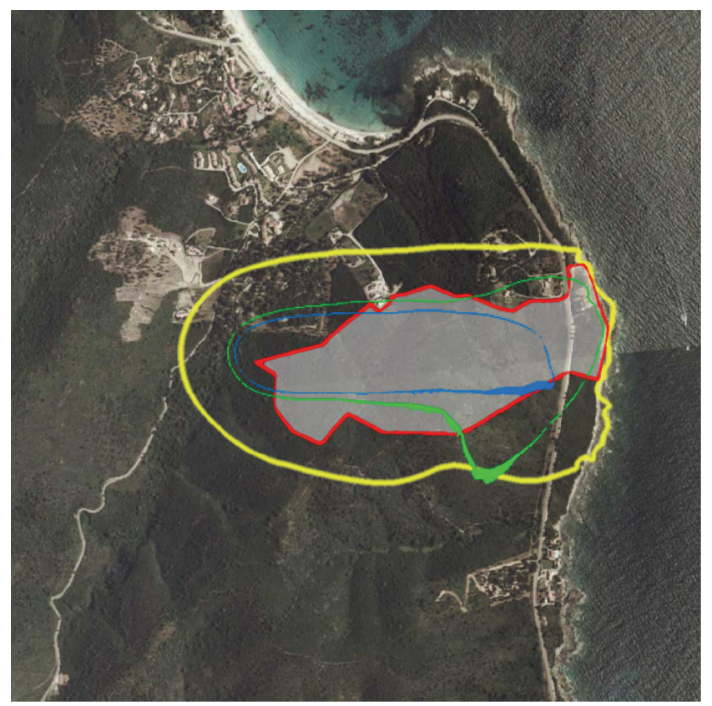

Figure 14: Case 2: Simulations results and observations for the Favone fire. Blue: simulated fire contour at 15:50 (after 50" ), Green: simulated contour at 19:00, yellow: simulated contour at 19:00 (non coupled); Red: final observed contour of the fire.

the online chemistry module of Meso- $\mathrm{NH}$ to investigate fire smoke and particle transport and validation with LIDAR measurements. 


\section{Acknowledgments}

Authors wish to thank the MesoNH team that helped developed the coupling. This research is developed within the IDEA project ANR-09-COSI-006-01. Field data was compiled in the scope of project PROTERINA-C supported by the EU under the Thematic 3 of the Operational Program Italia/France Maritime 2007-2013, Contract no. G25I08000120007.

\section{References}

[1] H. E. Graham, "Fire whirlwinds," Bulletin of the American Meteorological Society, vol. 36, no. 3, pp. 99-103, 1955.

[2] W. Mell, M. A. Jenkins, J. Gould, and P. Cheney, "A physicsbased approach to modelling grassland fires," International Journal of Wildland Fire, vol. 16, no. 1, pp. 1-22, 2007.

[3] N. P. Cheney and J. S. Gould, "Fire growth in grassland fuels," International Journal of Wildland Fire, vol. 5, no. 4, pp. 237347, 1995.

[4] R. Linn, J. Reisner, J. J. Colman, and J. Winterkamp, "Studying wildfire behavior using FIRETEC," International Journal of Wildland Fire, vol. 11, no. 3-4, pp. 233-246, 2002.

[5] W. E. Heilman and J. D. Fast, "Simulations of horizontal roll vortex development above lines of extreme surface heating," International Journal of Wildland Fire, vol. 2, pp. 55-68, 1992.

[6] R. Rothermel, "A mathematical model for predicting fire spread in wildland fuels," Research Paper INT-115, USDA Forest Service, 1972.

[7] T. L. Clark, J. Coen, and D. Latham, "Description of a coupled atmosphere-fire model," International Journal of Wildland Fire, vol. 13, no. 1, pp. 49-63, 2004.

[8] W. C. Skamarock and J. B. Klemp, "A time-split nonhydrostatic atmospheric model for weather research and forecasting applications," Journal of Computational Physics, vol. 227, no. 7, pp. 3465-3485, 2008.

[9] J. H. Balbi, F. Morandini, X. Silvani, J. B. Filippi, and F. Rinieri, "A physical model for wildland fires," Combustion and Flame, vol. 156, no. 12, pp. 2217-2230, 2009.

[10] J. P. Lafore, J. Stein, N. Asencio et al., "The Meso-NH Atmospheric Simulation System. Part I: adiabatic formulation and control simulations," Annales Geophysicae, vol. 16, no. 1, pp. 90-109, 1998.

[11] J. B. Filippi, F. Morandini, J. H. Balbi, and D. R. Hill, "Discrete event front-tracking simulation of a physical firespread model," Simulation, vol. 86, no. 10, pp. 629-644, 2010.

[12] J. Cuxart, P. Bougeault, and J. L. Redelsperger, "A turbulence scheme allowing for mesoscale and large-eddy simulations," Quarterly Journal of the Royal Meteorological Society, vol. 126, no. 562, pp. 1-30, 2000.

[13] P. Colella and P. R. Woodward, "The Piecewise Parabolic Method (PPM) for gas-dynamical simulations," Journal of Computational Physics, vol. 54, no. 1, pp. 174-201, 1984.

[14] R. Linn, J. Winterkamp, C. Edminster, J. J. Colman, and W. S. Smith, "Coupled influences of topography and wind on wildland fire behaviour," International Journal of Wildland Fire, vol. 16, no. 2, pp. 183-195, 2007.

[15] P. A. Santoni, A. Simeoni, J. L. Rossi et al., "Instrumentation of wildland fire: characterisation of a fire spreading through a Mediterranean shrub," Fire Safety Journal, vol. 41, no. 3, pp. 171-184, 2006. 

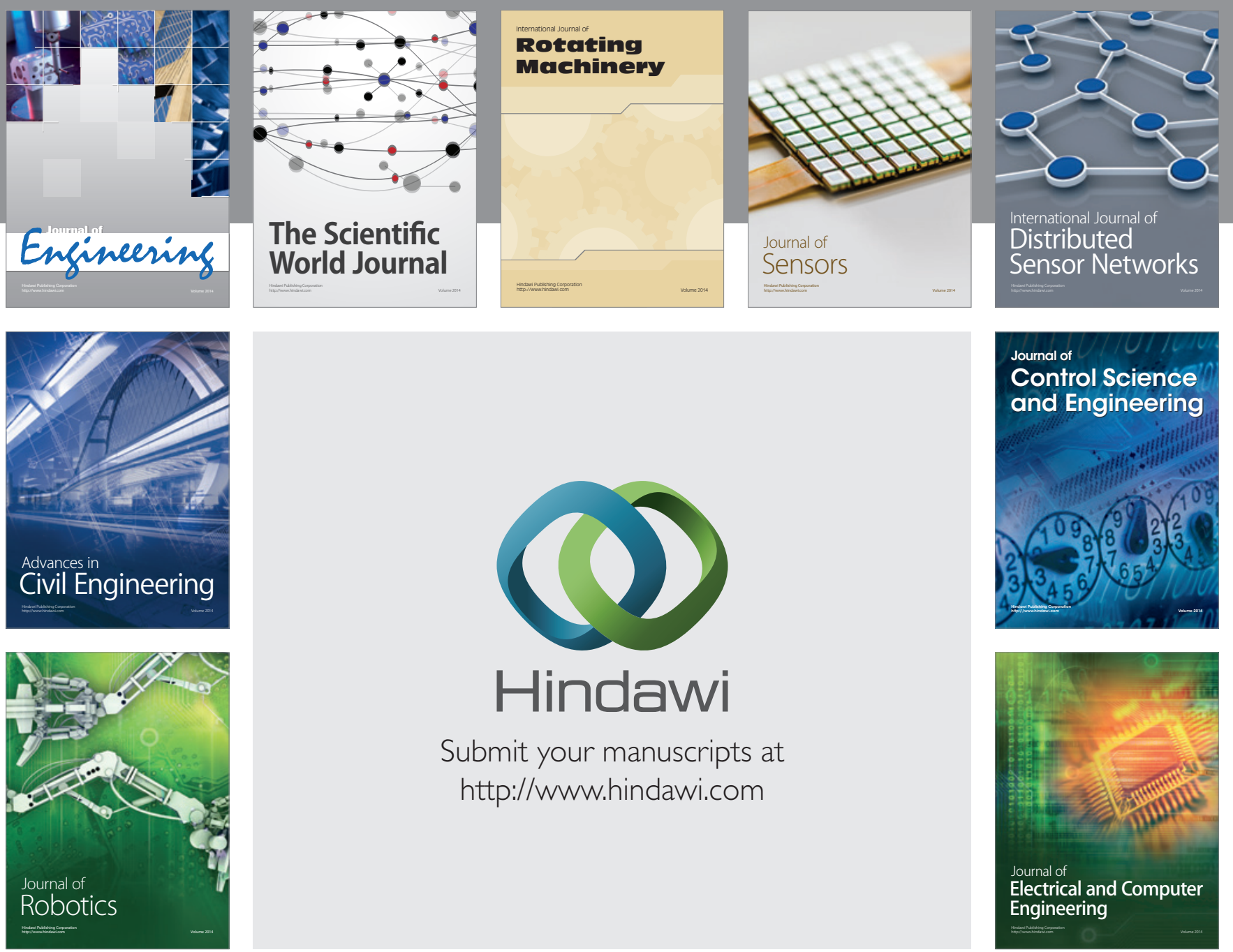

Submit your manuscripts at

http://www.hindawi.com
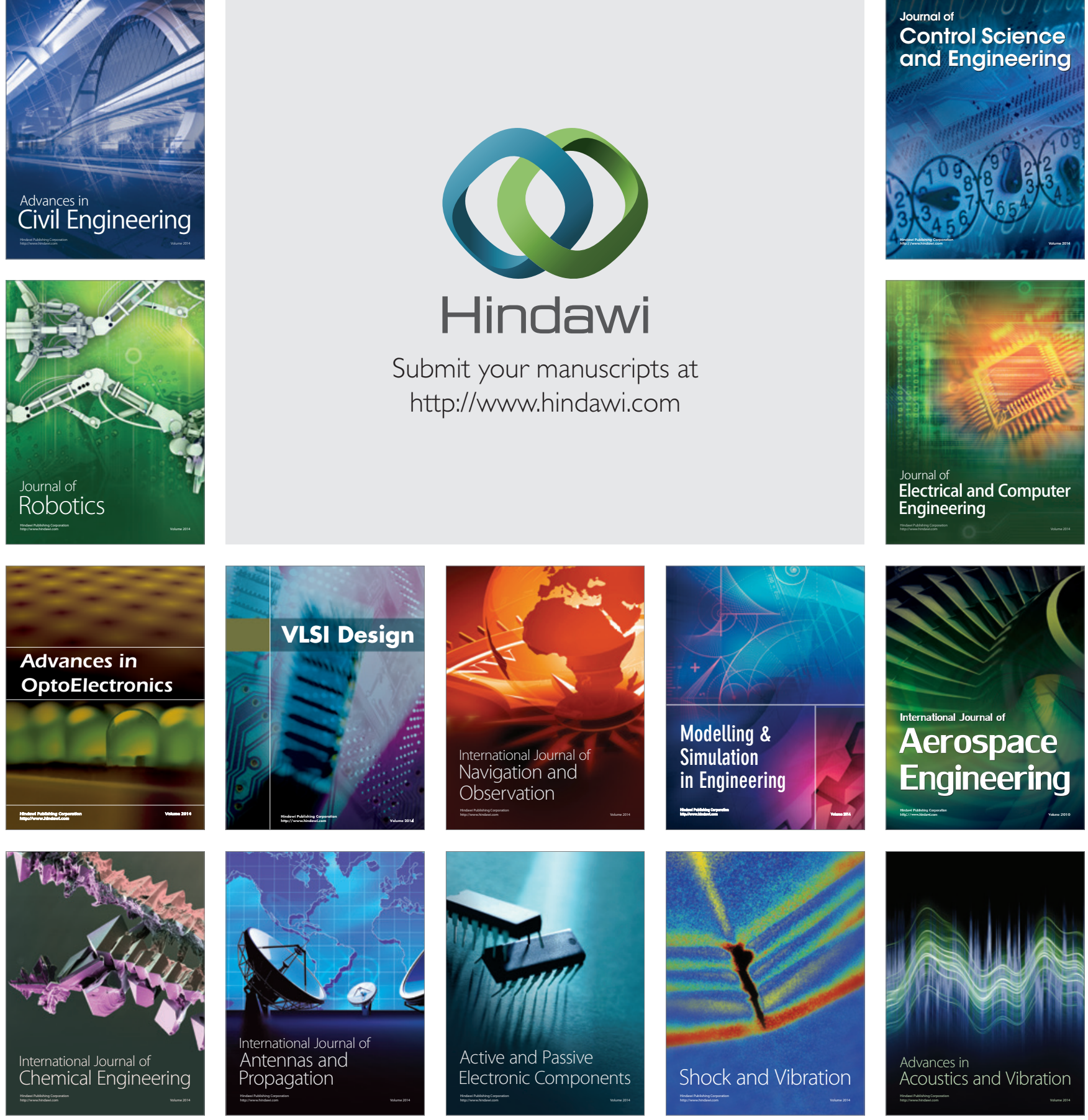